\title{
Eliminating behavior with omission and extinction after varying amounts of training*
}

\author{
CHARLES N. UHL \\ C'niversity of Ltah. Salt Lake Cit, Ltah 84112
}

\begin{abstract}
After rats were trained to leverpress for 1,3.9. or 27 day's on a variable interval reinforcement schedule. omission training was compared with extinction in effectiveness of response elimination. Extinction produced faster response elimination than omission, although both procedures eventually led to equal response elimination. Resistance to response elimination increased with length of baseline training. although this effect did not interact with omission vs extinction. A test of the durability of elimination effects followed. using a response-independent variable time reinforcement schedule. After extinction, resumption of responding in the durability test increased with length of baseline training. but there was little response resumption following omission regardless of the length of the baseline training. These results amplify and extend previous findings which show omission to be an effective and durable response elimination method.
\end{abstract}

Interest has increased recently in omission tiaining (Grant, 1964) as a means of eliminating behavior with reinforcement. Omission training is characterized by the postponement (omission) of a scheduled reinforcement event if a specified response occurs. Thus, reinforcement is received for not responding. Two temporal parameters operating in omission training are: (a) a response-reinforcement interval that sets the time each response delays reinforcement, and (b) a reinforcement-reinforcement interval that sets the time between consecutive reinforcements if no response intervenes. A more detailed discussion of these parameters and other characteristics of omission training is given in Uhl and Garcia (1969). In comparison with extinction or inild punishment, omission training is approximately as effective in eliminating responding, and its effects are more durable (Nevin, 1968: Topping. Pickering, \& Jackson. 1971a: Topping, Larmi. \& Johnson. 1972: Uhl \& Garcia. 1969; Uhl \& Sherman. 1971: Zeiler, 1971).

Now that the effectiveness of omission training has been demonstrated. there is need for investigations of its parameters. Topping. Pickering. and Jackson (1971a) have compared response elimination on omission schedules after VI or FI baseline training. They found that the reinforcement schedule used in baseline training did not interact with the effectiveness of response elimination on various omission schedules. This same result was obtained in this laboratory for comparisons of omission and extinction after VI or FI baseline training. Topping and his colleagues have also investigated FR schedules of reinforcement in baseline training prior to comparisons between omission and extinction (Topping. Pickering, \& Jackson. 1972). The FR reinforcement

*Supported by National Institute of Mental Health Research Grant 3H -139044 . Requests for reprints should be sent to Charles X. Lhl. Department of Psychology. L'niversity of Ltah. Sait Lake City. Ltah 84112. schedules did not interact with the relative effectiveness of omission vs extinction in this study. However, Topping, Pickering, and Jackson (1971b) did find that a differential reinforcement of low rates (DRL) schedule in baseline training enhanced the effectiveness of omission more than extinction. The latter result is to be expected, since the pause-between-responses requirement of a DRL schedule resembles the omission schedule requirement.

The purpose of the present study was to compare the effectiveness of omission and extinction after varying amounts of VI baseline training. It is well known (e.g.. D’Amato. Schiff. \& Jagoda, 1962: Miles, 1956: Perin. 1942: Skinner, 1938; Williams, 1938) that resistance to extinction of a free operant is an increasing function of the amount of baseline training prior to extinction. However, none of these studies tested the durability of response elimination following the discontinuation of extinction. In the present study, rats were given 1.3.9. or 27 daily sessions of VI baseline training before undergoing response elimination on omission or extinction schedules. After a criterion of response elimination was met. the durability of response elimination was assessed using a variable time (VT) schedule of response-independent reinforcement. A VT schedule was chosen for the durability test. since it constitutes a procedure identical to baseline training. except for the removal of a response requirement. which promotes resumption of responding (Uhl \& Garial. 1969: Lhl \& Sherman. 1971) even though there are no programmed consequences for responding.

\section{METHOD}

\section{Subjects}

The Ss were 32 experinentally naive male albino rats wejghing 250.300 g. The were maintained in individual cages at $80^{-}$of base weight throughout the course of the experiment. 
Table 1

Responses Per Minute for Each Group on the Last Day of Baseline

\begin{tabular}{rrrrrr}
\hline & \multicolumn{2}{c}{ Onmsion } & & \multicolumn{2}{c}{ Huinction } \\
\cline { 3 - 5 } Day & Mean & SD & & Mean & SD \\
\hline 27 & 18.50 & 5.83 & & 14.04 & 5.23 \\
9 & 15.54 & 2.41 & & 20.94 & 7.42 \\
3 & 9.22 & 2.47 & 12.70 & 7.82 \\
1 & 3.13 & 2.30 & 6.38 & 1.33 \\
\hline
\end{tabular}

Table 2

Days to Criterion for Each Group

\begin{tabular}{ccccrr} 
& \multicolumn{2}{c}{ Omission } & & \multicolumn{2}{c}{ Extinction } \\
\cline { 2 - 3 } \cline { 5 - 6 } Days & Mean & SD & & Mean & SD \\
\hline 27 & 13.75 & .43 & 13.00 & 1.73 \\
9 & 12.00 & 1.73 & 12.50 & .87 \\
3 & 8.25 & 1.30 & 8.75 & 1.78 \\
1 & 6.50 & 1.12 & 5.75 & .83 \\
\hline
\end{tabular}

\section{Apparatus}

Two identical operant boxes. each equipped with a liquid dipper $(.04 \mathrm{ml}$ cups). a retractable lever, an overhead houselight. and a speaker providing $85 \mathrm{~dB}$ white noise, served as test chambers A NOVA (Data General Corporation) computer operating a relay interface controlled experimental events, recorded responses. and provided data summary and analysis. The computer serviced each box once every $10 \mathrm{msec}$ to time experimental events and to monitor leverpresses.

\section{Procedure}

Lpon arrival in the laboratory. four rats were assigned randomly to each cell of a 2 by 4 factorial design incorporating estinction or omission training and 1.3. 9. or 27 days of baseline training, respectively. The start of the experiment was staggered for each group so that all rats began elimination training on the same day. After free-feeding base weight was obtained, the rats were handled in pairs for 5 days while their weight was reduced to $80^{\circ}$, of base weight. Magazine training, consisting of a VT 30-sec schedule, occurred on the following three daily 30-min sessions.

The positive reinforcer used throughout the experiment was a 30: (by weight) sucrose solution made available for $2 \mathrm{sec}$. On the day following magazine training. rats were shaped to press the lever and then were permitted 20 reinforcements on a fixed ratio (FR) 1 schedule followed by 20 reinforcements each on F R 2. FR 3. and FR 4.

After this preliminary training, the rats were given baseline training for daily $30-\mathrm{min}$ sessions on a variable interval (VI) 30-sec schedule for 1. 3. 9. or 27 day's. They were then placed on either extinction or omission. The reinforcement mechanism ceased to operate in extinction. Omission rats received reinforcement every $20 \mathrm{sec}$ if no response occurred, and a response delayed reinforcement for $40 \mathrm{sec}$. These parameter values were chosen to approximate those found by Lhl and Garcia $(1969)$ to be the most effective for response elimination. Each rat was run until it reached a criterion of no responses for a 10 -min period.

On the da! after reaching the elimination criterion. the rat was placed on a VT $30-$ sec schedule for six daily 30 -min sessions to test the durability of response elimination.

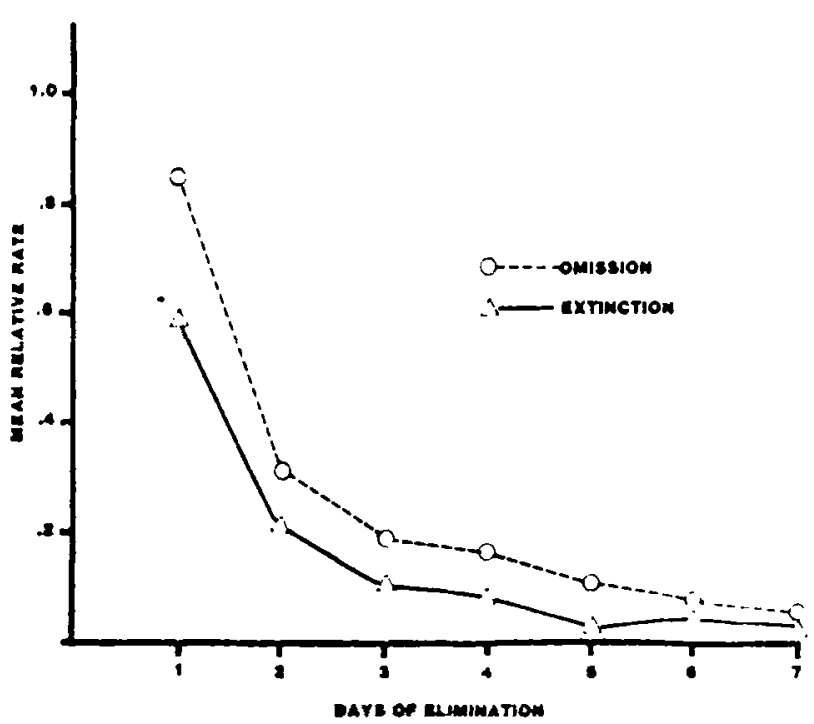

Fig. 1. Mean relative rate as a function of the first 7 days of elimination training with omission vs extinction as the parameter.

\section{RESULTS AND DISCUSSION}

Because of the inevitable differences in response rate after varying amounts of training $(F=10.18, d f=3 / 24$, $\mathrm{p}<.001$ ), all response rate data reported here were transformed by the shape function method proposed by Anderson (1963) to obtain a measure of relative rate. The transformation treated an individual rat's total responses in a session as a proportion of its total responses in the final session of baseline training, i.e., a unitary relative rate represents responding at the same rate as at the end of baseline training, and a zero relative rate represents cessation of responding. The mean and standard deviation of response rate for each group during the last day of baseline training is presented in Table 1 .

Number of days to reach the response elimination criterion was entered into a 2 by 4 analysis of variance incorporating omission vs extinction and amount of baseline training, respectively. Table 2 gives the mean and standard deviation for each group. No difference was found between omission and extinction ( $F<1$, $\mathrm{df}=1 / 24$ ), showing that both procedures resulted in the same degree of response elimination. The length of time necessary to achieve the elimination criterion increased with amount of baseline training $(F=39.07, d f=3 / 24$, $\mathrm{p}<.01$ ).

Although omission and extinction did not differ in time to produce response elimination. the question remains whether or not these procedures differed in the rate at which they reduced responding earlier in the elimination phase. To answer this question. relative 


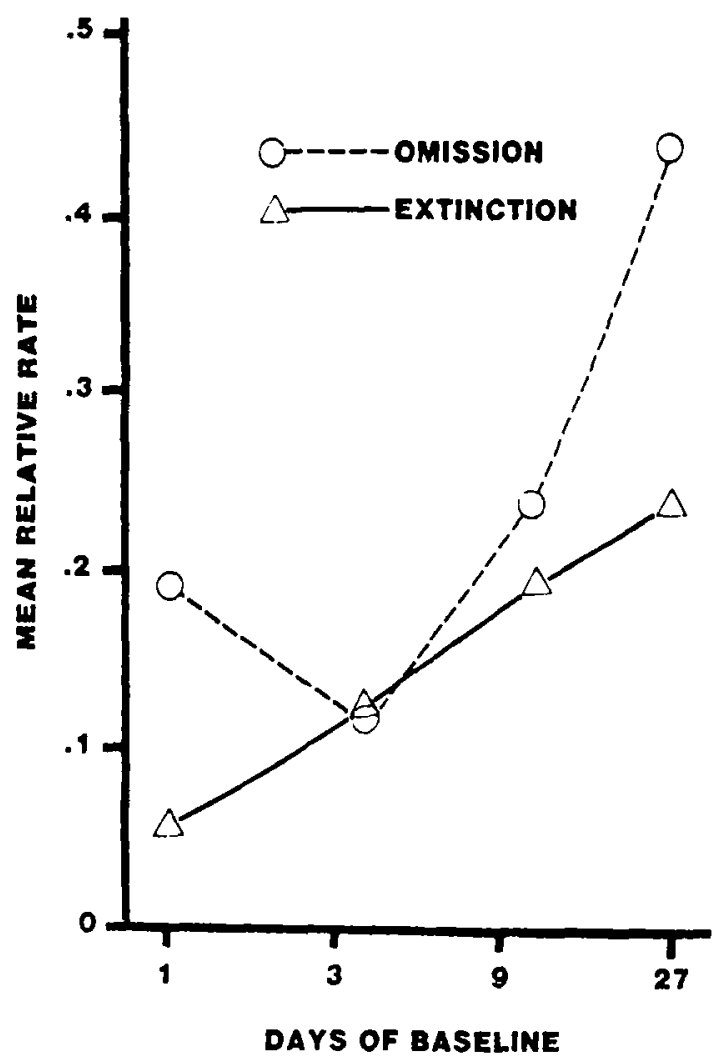

Fig. 2. Mean relative rate during elimination as a function of amount of baseline training with omission vs extinction as the parameter.

response rates were entered into a 2 by 4 by 7 analysis of variance incorporating omission vs extinction, amount of baseline training, and the first 7 days of elimination. (Seven days were selected since all but six rats had not yet reached criterion. A relative rate of zero was recorded for these rats on their postcriterion days.) Mean relative rate for omission vs extinction as a function of days of elimination is shown in Fig. 1. More rapid reduction in responding with extinction than with omission was reflected in the linear component of the interaction of omission vs extinction and days of elimination $(\mathrm{F}=8.11, \mathrm{df}=1 / 144, \mathrm{p}<.01)$. The linearity of this interaction was due to the diminishing difference between omission and extinction as daily sessions progressed.

These results confirm other findings (e.g., Uhl \& Garcia, 1969; Uhl \& Sherman, 1971) that response elimination is slower with omission than with extinction, although both procedures result in the same eventual amount of response elimination. Uhl and Garcia (1969) presented evidence indicating that omission effects are retarded by the discriminative stimulus control that the reinforcement event exerts over responding, e.g., reinforcement is discriminative for resumption of responding, especially at the beginning of omission training. The present finding of slower response elimination with omission can be accounted for by the same discriminative reinforcement interpretation.

The effect of amount of baseline training on response elimination is presented in Fig. 2. Response elimination was impeded by increasing amounts of baseline training (linear $F=24.92, \quad \mathrm{df}=1 / 24, \quad \mathrm{p}<.001$ ), a result confirming previous extinction studies. The interaction between omission vs extinction and amount of baseline training was not significant $(F=1.92, d f=3 / 24$, $\mathrm{p}<.10)$. This important finding indicates that response elimination with omission or extinction is affected equivalently by amount of baseline training. Other studies (Topping et al, 1971a, 1972) have shown that the reinforcement schedule used in baseline training does not interact with extinction vs omission comparisons, and the present results reveal that extinction vs omission comparisons are independent of length of baseline training.

The results of the VT durability test are shown in Fig. 3. Relative response rates were entered into a 2 by 4 by 6 analysis of variance incorporating omission vs extinction, amount of baseline training, and daily sessions of durability testing, respectively. The important outcome of this analysis was a significant linear component of the interaction between omission vs extinction and amount of baseline training $(F=14.83$, $\mathrm{df}=1 / 24, \mathrm{P}<.001$ ). This interaction can be seen in Fig. 3 if comparisons of responding are made between

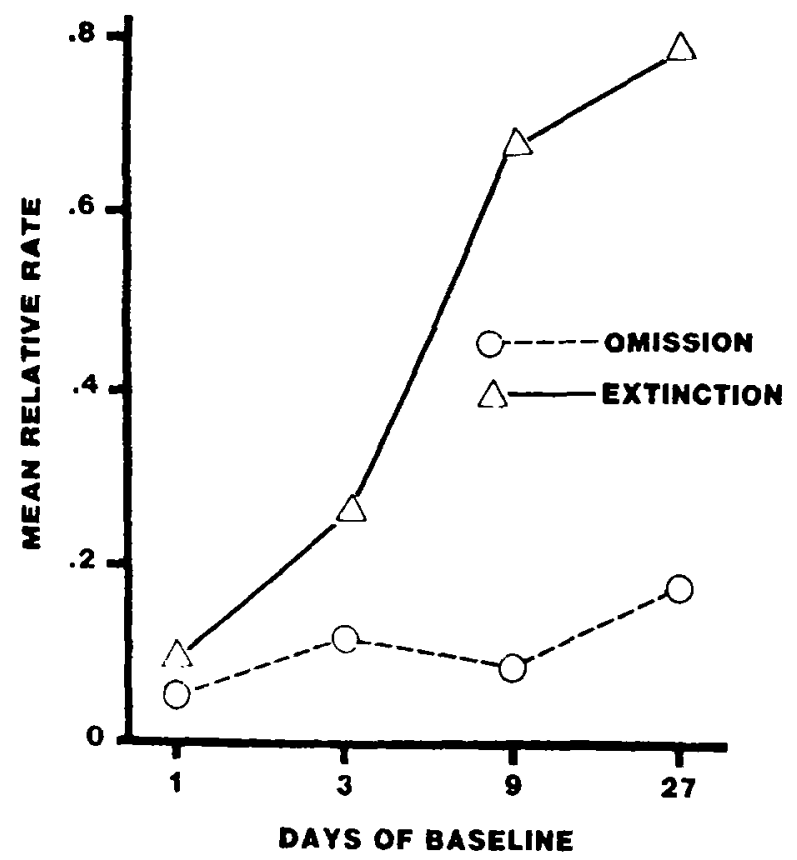

Fig. 3. Mean relative rate during the durability test as a function of amount of baseline training with omission vs extinction as the parameter. 
omission and extinction groups as a function of increasing amounts of baseline training. Responding in the omission groups remained at the same low level while it increased greatly in the extinction groups. This result confirms and extends previous findings (Uhl \& Garcia, 1969: Uhl \& Sherman. 1971; Zeiler, 1971) of greater durability of response elimination following omission in comparison with extinction. The present results show that response elimination after omission is highly durable regardless of the amount of baseline training. In contrast. however, extinction effects are increasingly less durable as the amount of baseline training increases. When extinction is discontinued and replaced by a procedure resembling baseline training, responding resumes at substantial rates.

The considerable amounts of response resumption in the VT durability test after extinction probably were due to the discriminative effects of the reinforcement event itself. Similar findings have been reported by Reid (1957) and by Rescorla and Skucy (1969). The discriminative properties of reinforcement are largely unchanged during extinction since reinforcement never occurs. When reinforcement is reintroduced in the VT durability test following extinction, the discriminative control of the reinforcement event reasserts itself and leads to resumption of responding. In fact, there is evidence that reinforcement becomes discriminative for not responding during omission training (Uhl \& Garcia, 1969). It is to be expected, then, that reinforcements provided by the VT schedule in the durability test should not bring about response resumption. It is particularly impressive that omission effects remained durable even in the case of Ss given 27 days of baseline training.

\section{REFERENCES}

Anderson. N. H. Comparison of different populations: Resistance to extinction and transfer. Psychological Review, 1963, 70. 162-179.

D'Amato. M. R., Schiff, D., \& Jagoda, H. Resistance to extinction after varying amounts of discriminative or nondiscriminative instrumental training. Journal of Experimental Psychology, 1962, 64, 526-532.
Grant. D. A. Classical and operant conditioning. In W. A. Melton (Ed.). Categories of human learning. New York: Academic Press, 1964.

Miles. R. C. The relative effectiveness of secondary reinforcers throughout deprivation and habit-strength parameters. Journal of Comparative \& Physiological Psychology. 1956. 49. 126-130.

Nevin. I. A. Differential reinforcement and stimulus control of not responding. Journal of the Experimental Analysis of Behavior, 1968, 11, 715-726.

Perin, C. T. Behavior potentiality as a joint function of amount of training and degree of hunger at the time of extinction. Journal of Experimental Psychology, 1942, 30, 93-113.

Reid. R. L. The role of the reinforcer as a stimulus. British Journal of Psychology, 1957, 49, 202-209.

Rescorla, R. A., \& Skucy, J. C. Effect of response independent reinforcers during extinction. Journal of Comparative \& Physiological Psychology. 1969, 67, 381-389.

Skinner, B. F. The behavior of organisms. New York: Appleton-Century, 1938.

Topping. J. S., Larmi, O. K., \& Johnson, D. L. Omission training: Effects of gradual introduction. Psychonomic Science, 1972, 28, 279-280.

Topping, J. S., Pickering, J. W., \& Jackson, J. A. Omission training effects following VI and FI pretraining. Psychonomic Science, 1971a, 24, 113-114.

Topping, J. S., Pickering, J. W., \& Jackson, J. A. The differential effects of omission and extinction following DRL pretraining. Psychonomic Science, 1971b, 24, 137-138.

Topping. J. S., Pickering, J. W., \& Jackson, J. A. Comparison of omission and extinction following FR reinforcement training. Psychological Record, 1972, 22, 221-224.

Uhl, C. N., \& Garcia, E. E. Comparison of omission with extinction in response elimination in rats. Journal of Comparative \& Physiological Psychology, 1969, 69, 554-562.

Uhl, C. N., \& Sherman, W. O. Comparison of combination of omission, punishment, and extinction methods in response elimination in rats. Journal of Comparative \& Physiological Psychology, 1971, 74, 59-65.

Williams, S. B. Resistance to extinction as a function of the number of reinforcements. Journal of Experimental Psychology, 1938, 23, 506-522.

Zeiler, M. D. Eliminating behavior with reinforcement. Journal of the Experimental Analysis of Behavior, 1971, 16, 401-405.

(Received for publication January 1, 1973: revision received May 25, 1973; accepted June 25,1973 .) 